\section{REGULACIÓN DE LA EUTANASIA Y EL SUICIDIO ASISTIDO EN ESPAÑA ¿HACIA QUÉ MODELO SE DIRIGE LA OPINIÓN PÚBLICA?}

\author{
M.a Ángeles Molina Martínez \\ Consejo Superior de Investigaciones Científicas. \\ Instituto de Estudios Sociales Avanzados \\ mmolina@iesa.csic.es \\ Rafael Serrano-del-Rosal \\ Consejo Superior de Investigaciones Científicas. \\ Instituto de Estudios Sociales Avanzados \\ rserrano@iesa.csic.es
}

\begin{abstract}
Cómo citar este artículo/Citation: Molina Martínez, M.A. y Serrano-del-Rosal, R. (2014). "Regulación de la eutanasia y el suicidio asistido en España chacia qué modelo se dirige la opinión pública?". Arbor, 190 (769): a174. doi: http://dx.doi. org/10.3989/arbor.2014.769n5013
\end{abstract}

Recibido: 9 julio 2013. Aceptado: 3 febrero 2014.

RESUMEN:

Introducción: Comparar las actitudes de los ciudadanos españoles con los modelos legales existentes sobre eutanasia y suicidio asistido para identificar con cuál de ellos la ciudadanía podría estar más de acuerdo.

Metodología: Se analizaron los datos recogidos por el Centro de Investigaciones Sociológicas en el Estudio 2.803 "Atención para pacientes con enfermedad terminal". Realizamos análisis descriptivos y comparaciones mediante la prueba de McNemar. Resultados: La población española apoya claramente la práctica de eutanasia en enfermos terminales. El suicidio médico asistido y los casos de enfermedad degenerativa hacen disminuir el grado de aceptación.

Conclusiones: El modelo belga es el que mejor se ajusta a las opiniones de la ciudadanía española. Se considera un punto de partida, para conducir el debate social, político y jurídico, necesario para lograr una regulación de los derechos que asisten a los ciudadanos al final de la vida.

PALABRAS CLAVE: eutanasia; suicidio médicamente asistido; opinión pública; legislación.

\section{REGULATING EUTHANASIA AND ASSISSTED SUICIDE IN SPAIN. WHAT MODEL IS PUBLIC OPINION TENDING TOWARDS?}

Copyright: (c) 2014 CSIC. Este es un artículo de acceso abierto distribuido bajo los términos de la licencia Creative Commons Attribution-Non Commercial (by-nc) Spain 3.0.

\section{ABSTRACT:}

Objective: To compare the attitudes of Spanish citizens with existing legal models on euthanasia and assisted suicide to identify which of them the citizens could agree with.

Methods: Data collected by the Social Research Centre in Study 2,803 "care for patients who are terminally ill" in 2009 were analysed. Descriptive analysis and comparisons with McNemar test were carried out.

Results: Spanish populationclearly supports the practice of euthanasia for the terminally ill. Agreement is lower in the case of physician assisted suicide and degenerative diseases.

Conclusions: The Belgian model matches the approach favoured by Spanish public opinion closest. This represents a starting point for the social, political and legal debate needed to regulate individuals' rights at the end of life.

KEYWORDS: euthanasia; physician assisted suicide; public opinion; legislation. 


\section{INTRODUCCIÓN}

En la actualidad todos los estados Europeos activan políticas que se han venido a llamar de Welfare Mix, a saber, que deben ser participadas tanto por el Estado como por el mercado y los propios ciudadanos. Estas tienen como una de sus principales características una concepción de la ciudadanía que no se define ya como pertenencia del individuo al Estado sino más bien como complejo de derecho-deberes de los "sujetos de ciudadanía" (Herrera, 2001). Todo ello entraña, entre otras cosas, que las políticas no se diseñen para los ciudadanos sino más bien con los ciudadanos, puesto que el bienestar debe de ser expresión de la sociedad más que del Estado. En el ámbito de las políticas de salud, sabemos que para analizar por ejemplo la calidad de un servicio sanitario es fundamental contar también con instrumentos que recaben e integren la opinión y satisfacción de sus usuarios (Ministerio de Sanidad, Política Social e Igualdad, 2010). Es sobre estos argumentos, sobradamente conocidos por otra parte, sobre los que descansa la idea de la centralidad del ciudadano en el proceso de diseño, implementación, evaluación comprensiva y rediseño de una determinada política pública, más aún en el ámbito de la salud.

En el caso que nos ocupa el interés radica en analizar la opinión ciudadana sobre dos cuestiones controvertidas como son la eutanasia y el suicidio asistido, para que el debate que se está produciendo desde hace años en España -con desigual presencia social y mediática en distintos momentos-sobre lo que genéricamente se conoce como derechos ciudadanos en el proceso final de la vida o "derechos de salida", como gusta decir a M. a Ángeles Durán (2007), no se haga precisamente al margen de la ciudadanía, sino integrando también sus opiniones y actitudes. Partimos de tres hechos sociales, empíricamente contrastados y fundamentales para el análisis de este fenómeno:1) según los resultados de diversas encuestas (Durán, 2004; de Miguel y López, 2006; Instituto de Estudios Sociales Avanzados (IESA), 2007; Centro de Investigaciones Sociológicas (CIS), estudio 2.803, "Atención a pacientes con enfermedad en fase terminal”, 2009), la ciudadanía española apoya mayoritariamente la regulación de la eutanasia y del suicidio médico asistido (SMA).2)Entre aquellos países que han regulado estas prácticas, no existen dos legislaciones idénticas (Tomás-Valiente, 2005; Royes, 2008).3) No tenemos información empírica de ningún tipo que aporte conocimiento sobre el modelo de regulación que desearía la ciuda- danía española ni tampoco análisis sobre el modelo de regulación que pudiera derivarse de sus opiniones y actitudes. Son estos hechos los que nos llevan a plantear el objetivo principal de esta investigación: analizar las opiniones y actitudes de la ciudadanía española con objeto de conocer empíricamente qué modelo de regulación de la eutanasia y/o suicidio asistido (SA) de los que actualmente existen en los países de nuestro entorno estaría más en consonancia con sus opiniones y actitudes o si, por el contrario, ninguno de los desarrollados da cobertura a los intereses de la ciudadanía española. Ello permitirá aportar una información nueva, relevante en las sociedades del bienestar, y de base empírica, al controvertido debate sobre el proceso final de la vida y los derechos que deben asistir al ciudadano en este proceso, debate que las más de las veces se hace de espaldas a los propios ciudadanos.

El marco analítico que se utilizará como referencia lo conforman las tres situaciones legales que podemos encontrar en las sociedades avanzadas contemporáneas en la actualidad:

1a) Prohibición de la eutanasia y el suicidio asistido. La mayoría de los países, incluido España, consideran la eutanasia un delito, sancionándola en el amplio marco "de las conductas de colaboración en el suicidio de otra persona y/o de ejecución de un homicidio con la voluntad de la víctima" (TomásValiente, 2005). En concreto, la legislación española sanciona esta práctica con penas de cárcel pero contempla atenuantes en los casos donde la víctima sufre una enfermedad que le conduce a la muerte o a un sufrimiento permanente y difícil de soportar (Ley №10/1995 de 23 de noviembre de 1995).

2a) Legalización solo de la eutanasia o solo del suicidio asistido. En este segundo bloque podemos encontrar las regulaciones de diferentes Estados.

a) El artículo 115 del Código Penal suizo (RS 311.0 de 21 de diciembre de 1937) condena a aquel que por un motivo egoísta incite a una persona a cometer suicidio o asista al suicidio de un tercero. En Suiza por tanto no se regula legalmente el suicidio asistido, ni se controla en el marco del sistema sanitario; más bien se considera que este no es un delito punible, a condición de que se realice por motivos desinteresados.

b) El Estado de Oregón (Rev. Stat. 127.800-.897 de 27 de octubre de 1997), permite a los profesionales de la Medicina prescribir la dosis adecuada de medicación para que sus pacientes puedan poner 
fin a su vida, si se cumplen una serie de condiciones: que el enfermo sea adulto, que padezca una enfermedad terminal, que sea plenamente capaz, que haya expresado voluntariamente su deseo de morir, que haya formulado su petición por escrito y que resida en Oregón.

c) Washington también ha legalizado el SMA en condiciones semejantes al Estado de Oregón y, en Montana, distintas resoluciones judiciales han dejado sin penalizar la ayuda al suicidio en el caso de enfermos terminales.

d) Bélgica (parlementaires du Sénat de Belgique 2001-2002) legaliza la eutanasia, aunque no el suicidio asistido, bajo las siguientes condiciones:

i) El paciente ha de ser mayor de edad o menor emancipado, capaz y consciente en el momento de la solicitud de eutanasia.

ii) La petición debe formularse de manera voluntaria, reflexionada y repetida y no debe ser resultado de presiones externas.

iii) Ha de ser registrada por escrito y debe transcurrir al menos un mes hasta la práctica de la eutanasia.

iv) El paciente ha de encontrarse en una situación médica sin esperanza y bajo un sufrimiento físico o psicológico constante e insoportable que no puede ser apaciguado, resultado de una condición accidental o patológica grave e incurable.

v) El médico ha de informar al paciente sobre su estado y pronóstico y discutir con él las posibles alternativas llegando ambos a la conclusión de que no hay alternativa razonable.

vi) Debe consultarse a un médico independiente, quien examinará al enfermo, consultará su historial médico y asegurará el cumplimiento de la tercera condición.

vii) En el caso de que no se trate claramente de una enfermedad terminal, deberá ser consultado un tercer médico.

3a) Legalización tanto de la eutanasia como del suicidio asistido. En este tercer grupo podemos identificar solo dos países, ambos europeos.

a) En los Países Bajos (Act. Senate, session 20002001, 26 691, number 137), la Ley permite y regula la eutanasia y el SMA, bajo el cumplimiento de los siguientes supuestos: i) Debe existir una petición voluntaria y reflexionada por parte del paciente.

ii) Ha de encontrarse en una situación de sufrimiento insoportable.

iii) Debe ser informado acerca de la situación en la que se encuentra y de sus perspectivas.

iv) Médico y paciente han de llegar juntos a la conclusión de que no existe otra solución más satisfactoria.

v) Un médico independiente debe examinar al paciente y elaborar un informe escrito en el que corrobore el cumplimiento de los anteriores criterios.

vi) El médico debe llevar a cabo la eutanasia o el SMA con el cuidado debido.

b) Luxemburgo (Memorial Journal Officiel du GrandDuché de Luxembourg, 2009) regula y despenaliza la eutanasia y el SMA en los siguientes casos:

i) El paciente es adulto, capaz y consciente en el momento de la solicitud.

ii) La petición es voluntaria, reflexionada, repetida y no es resultado de presiones externas.

iii) El paciente se encuentra en una situación médica terminal, con sufrimiento constante e insoportable, y sin perspectivas de mejora.

iv) La solicitud debe hacerse por escrito.

v) El médico deberá informar al paciente de su estado de salud y de sus perspectivas, así como de las posibilidades terapéuticas y paliativas existentes y contar con la opinión de un segundo experto.

Los modelos de legislación presentados de forma concisa (ya sean solo de eutanasia o SA, ya de ambos) tienen en común, reconocer el ámbito sanitario como espacio donde se desarrollan, controlan y ejecutan estos procesos, excepto el caso suizo en el que más que una regulación lo que se da es una no penalización punitiva. Otra característica importante y compartida por los tres países europeos cuya regulación es más explícita (Países Bajos, Bélgica y Luxemburgo) es que reconocen la validez del testamento vital también como petición de fin de la vida bajo los supuestos recogidos en sus respectivas leyes. En cuanto a los elementos más importantes que diferencian las regulaciones expuestas podemos señalar tres: 
1) Las diversas posibilidades de despenalización. En el caso de Suiza dentro del Código Penal, se establecen espacios de impunidad que permiten la colaboración con el suicidio de otra persona, lo cual no es una regulación explícita. El resto de países han promulgado leyes regulando las acciones que tienen por objetivo poner fin a la vida.

2) Las prácticas legalizadas. Suiza, Oregón y Washington, despenalizan el SA, pero solo Suiza lo permite sin que se produzca bajo el control del sistema sanitario; Bélgica solo permite la eutanasia y los Países Bajos y Luxemburgo ambas prácticas.

3) Las condiciones bajo las cuales se pueden practicar la eutanasia y/o el SA. En Suiza el único requisito es que no existan "motivos egoístas"; el paciente ha de encontrarse en una situación de sufrimiento insoportable en los Países Bajos y padecer una enfermedad en fase terminal en los casos de Oregón, Washington, Luxemburgo y Bélgica (donde se contemplan otros casos supeditados al criterio de un tercer médico).

En base a estas diferencias fundamentales, analizaremos la opinión de los ciudadanos españoles con objeto de derivar de dicho análisis el modelo de regulación que mejor acomodaría las opiniones y actitudes de la ciudadanía en este momento, lo cual centraría y orientaría el necesario debate social, político y legal e incluso facilitaría la consecución de un debate de base empírica sobre las consecuencias de este tipo de regulación.

\section{MATERIAL Y MÉTODOS}

Para el objetivo empírico de este trabajo hemos utilizado parte de los datos recogidos por el CIS en el Estudio CIS 2.803, "Atención a pacientes con enfermedad en fase terminal", del año 2009. Se entrevistó a una muestra representativa de la población nacional, formada por 2.481 personas (48.1\% varones), de 18 años y más. La ficha técnica del estudio y su matriz de datos y diferentes tabulaciones están disponibles en: http://www.cis.es/cis/opencm/ES/1_encuestas/ estudios/ver.jsp?estudio $=9982$

Del cuestionario original seleccionamos aquellas preguntas (ver Tabla I) que permiten analizar la actitud de los ciudadanos respecto a las principales diferencias que hemos señalado que existen entre

Tabla I. Selección de preguntas

\begin{tabular}{|c|c|}
\hline Diferencias fundamentales entre legislaciones & Preguntas seleccionadas \\
\hline \multirow[t]{2}{*}{ Posibilidades de regulación } & $\begin{array}{l}\text { P.24 Cuando una persona tiene una enfermedad en fase terminal, que le } \\
\text { causa grandes sufrimientos y que le causará la muerte en poco tiempo, } \\
\text { ¿cree Ud. que la ley debería permitir que los médicos pudieran poner fin a } \\
\text { su vida y a sus sufrimientos, si esta persona lo solicita libremente? }\end{array}$ \\
\hline & $\begin{array}{l}\text { P.28 ¿Cree Ud. que debería castigarse al médico que ponga fin, sin dolor, } \\
\text { a la vida de un paciente, en la fase final de una enfermedad irreversible si } \\
\text { éste se lo pide de manera reiterada y consciente? }\end{array}$ \\
\hline \multirow[b]{2}{*}{ Prácticas legalizables } & P.37 ¿Cree Ud. que en España debería regularse por Ley la eutanasia? \\
\hline & $\begin{array}{l}\text { P.38 (cree Ud. que en España debería regularse por Ley) ¿Y el suicidio mé- } \\
\text { dicamente asistido? }\end{array}$ \\
\hline \multirow{3}{*}{ Condiciones exigidas } & $\begin{array}{l}\text { P.25 (enfermedad en fase terminal) Y si esta persona lo solicita libremente a } \\
\text { sus médicos, ¿cree Ud. que la ley debería permitir que éstos le proporcionasen } \\
\text { los medios necesarios para que ella misma pudiera terminar con su vida? }\end{array}$ \\
\hline & $\begin{array}{l}\text { P.35 En el caso de una persona mayor de } 18 \text { años, con una enfermedad } \\
\text { degenerativa incurable, que le va a incapacitar mental y físicamente en un } \\
\text { futuro próximo, ¿piensa Ud. que si lo ha solicitado, la ley debería autorizar } \\
\text { a los médicos a terminar con su vida cuando ella lo decida? }\end{array}$ \\
\hline & $\begin{array}{l}\text { P.36 (persona mayor de } 18 \text { años, con una enfermedad degenerativa incu- } \\
\text { rable) Y si esta persona lo solicita, ¿cree que la ley debería autorizar a los } \\
\text { médicos a recetarle una dosis suficiente de fármacos para que ella misma } \\
\text { termine con su vida, tomándoselos cuando lo decida? }\end{array}$ \\
\hline
\end{tabular}

Fuente: Centro de Investigaciones Sociológicas, España, 2009. Encuesta nacional, 2.481 entrevistas a población mayor de 18 años. Número de Estudio: 2.803. 
las legislaciones contempladas: 1) tipo de regulación (legislar vs no sancionar), preguntas P24 y P28; 2) legalización de la eutanasia y el SMA, P37 y P38; y 3) supuestos bajo los que se permite llevar a cabo estas prácticas P25, P35 y P36.

Con el fin de conocer las opiniones y actitudes manifestadas por la ciudadanía al respecto de los temas que nos interesan en esta investigación y de conocer si dichas opiniones apoyan alguno de los marcos legislativos existentes en nuestro entorno político y económico, llevamos a cabo una serie de análisis descriptivos en aplicación del principio de simplicidad. En un primer momento analizamos los porcentajes de respuestas de las diferentes categorías de las variables utilizadas y la moda como medida de tendencia central adecuada al nivel de medición de las variables. Posteriormente, recodificamos las categorías de respuesta originales ("sí, con toda seguridad", "creo que sí, pero no estoy totalmente seguro/a" vs "creo que no, pero no estoy totalmente seguro/a", "no, con toda seguridad") convirtiendo la variable en dicotómica (acuerdo vs. desacuerdo) y analizamos cómo se distribuye el grado de acuerdo o desacuerdo general.

Hecho esto y con el fin de identificar cuáles son las situaciones en las que los ciudadanos alcanzan un alto grado de acuerdo sin indecisión y en cuáles existe mayor incertidumbre, se hizo una nueva recodificación en la que por un lado se daba cuenta de aquellas opiniones favorables tanto a la eutanasia como al SMA sin vacilación alguna, es decir, contemplando exclusivamente la categoría de respuesta "sí, con toda seguridad" y agrupando a todas las demás categorías de respuesta, incluida la categoría "creo que sí, pero no estoy totalmente seguro/a" en el grupo de aquellos que están en contra de estas situaciones. Con esta decisión metodológica se trataba de minimizar los falsos positivos, a sabiendas de que se han incrementado sin duda los falsos negativos, ya que en el grupo de los que se oponen se han incluido a aquellos que si bien están a favor muestran algún tipo de duda o reticencia. Con esta estrategia de análisis nos aseguramos que cuando más adelante se argumente sobre la cercanía de la opinión de los españoles a un modelo legislativo $u$ otro se haga con un respaldo empírico sólido, lo cual se consideró la estrategia más adecuada dada la importancia y dificultad de los temas abordados.

Por último, utilizando esta última recodificación, a la que denominamos grado de acuerdo sin ambigüedad, comprobamos si la proporción de personas que eligen esta categoría difiere de forma significativa en función de determinados supuestos. Para ello seleccionamos pares de preguntas y contrastamos la hipótesis de homogeneidad marginal (es decir, de igualdad de proporciones marginales) mediante la prueba de McNemar.

\section{RESULTADOS}

En primer lugar hay que recordar que todas las situaciones planteadas a los encuestados que se analizan, tratan de recabar su opinión sobre diferentes situaciones que pudieran acontecer en la etapa final de la vida y que tienen un objetivo común: poner fin a la vida del paciente por su petición explícita; si bien es verdad que bajo distintos supuestos: padecer una enfermedad terminal o degenerativa; Ilevando a cabo la acción un médico o el propio paciente, etc. Como se observa en la distribución de frecuencias de la Tabla II, en todas las variables las respuestas se concentran en las categorías que muestran una actitud positiva con los supuestos que se plantean. El valor modal en todas las respuestas corresponde a 1 "sí, con toda seguridad", salvo cuando se pregunta si un médico debe ser castigado por poner fin a la vida de un paciente (P.28), donde el valor modal fue 4 "no, con toda seguridad" respuesta que, en cualquier caso, va en la misma línea de los resultados anteriores, es decir, una postura modal favorable a lo que en general se ha denominado derechos de salida, a que se regulen y a que se practiquen en el ámbito sanitario. Las frecuencias que se concentran en el valor modal oscilan entre un $63.3 \%$ (supuesto de un médico que pone fin a la vida de un paciente con enfermedad en fase terminal, que le causa grandes sufrimientos y que le causará la muerte en poco tiempo, P.24) y un 38.9\% (cuando los ciudadanos opinan sobre la legalización del SMA, P.38). Analizando con mayor detenimiento el valor modal, comprobamos que la mitad o más de la muestra manifiesta su acuerdo con total seguridad en las siguientes situaciones: caso de eutanasia en un paciente con enfermedad terminal (P.24), no castigar al médico que lleve a cabo un acto de eutanasia en un paciente con una enfermedad irreversible (P.28) y legalizar la eutanasia en España (P.37). Y aun siendo el valor modal el estar de acuerdo "con toda seguridad", este es un valor modal inferior al $50 \%$ de la muestra, en los siguientes casos: el SMA en un paciente con enfermedad terminal (P.25), que sea el paciente con una enfermedad degenerativa incurable quien decida el momento en el que le sea practicada una eutanasia (P. 35); SMA en un paciente con una enfermedad degenerativa incurable (P.36) y legalizar el SMA (P.38). 
Tabla II. Descriptivos y medida de tendencia central

\begin{tabular}{|c|c|c|c|c|c|c|c|c|c|}
\hline & (1) & (2) & (3) & \multicolumn{2}{|c|}{ (4) } & (5) & (6) & Total & Moda \\
\hline $\begin{array}{l}\text { P.24 Cuando una persona tiene una enfermedad } \\
\text { en fase terminal, que le causa grandes sufrimien- } \\
\text { tos y que le causará la muerte en poco tiempo, } \\
\text { ¿cree Ud. que la ley debería permitir que los médi- } \\
\text { cos pudieran poner fin a su vida y a sus sufrimien- } \\
\text { tos, si esta persona lo solicita libremente? }\end{array}$ & $\begin{array}{l}\mathrm{N}: 1570 \\
63.3 \%\end{array}$ & $\begin{array}{l}\mathrm{N}: 426 \\
17.2 \%\end{array}$ & $\begin{array}{l}\mathrm{N}: 131 \\
5.3 \%\end{array}$ & \multicolumn{2}{|c|}{$9.2 \%$} & $\begin{array}{l}N: 104 \\
4.2 \%\end{array}$ & $\begin{array}{l}\mathrm{N}: 21 \\
0.8 \%\end{array}$ & $\begin{array}{c}N: 2481 \\
100 \%\end{array}$ & 1 \\
\hline $\begin{array}{l}\text { P. } 25 \text { Y si esta persona lo solicita libremente a sus } \\
\text { médicos, ¿cree Ud. que la ley debería permitir que } \\
\text { éstos le proporcionasen los medios necesarios } \\
\text { para que ella misma pudiera terminar con su vida? }\end{array}$ & $\begin{array}{c}\mathrm{N}: 1233 \\
49.7 \%\end{array}$ & $\begin{array}{l}N: 451 \\
18.2 \%\end{array}$ & $\begin{array}{l}\mathrm{N}: 225 \\
9.1 \%\end{array}$ & \multicolumn{2}{|c|}{$16.2 \%$} & $\begin{array}{l}N: 159 \\
6.4 \%\end{array}$ & $\begin{array}{l}\mathrm{N}: 12 \\
0.5 \%\end{array}$ & $\begin{array}{c}\mathrm{N}: 2481 \\
100 \%\end{array}$ & 1 \\
\hline $\begin{array}{l}\text { P.28 ¿Cree Ud. que debería castigarse al médico } \\
\text { que ponga fin, sin dolor, a la vida de un paciente, } \\
\text { en la fase final de una enfermedad irreversible si } \\
\text { éste se lo pide de manera reiterada y consciente? }\end{array}$ & $\begin{array}{l}\mathrm{N}: 182 \\
7.3 \%\end{array}$ & $\begin{array}{l}\mathrm{N}: 175 \\
7.1 \%\end{array}$ & $\begin{array}{l}N: 420 \\
16.9 \%\end{array}$ & \multicolumn{2}{|c|}{$58.8 \%$} & $\begin{array}{l}\mathrm{N}: 225 \\
9.1 \%\end{array}$ & $\begin{array}{l}\mathrm{N}: 19 \\
0.8 \%\end{array}$ & $\begin{array}{c}N: 2481 \\
100 \%\end{array}$ & 4 \\
\hline $\begin{array}{l}\text { P.35 En el caso de una persona mayor de } 18 \text { años, } \\
\text { con una enfermedad degenerativa incurable, que } \\
\text { le va a incapacitar mental y físicamente en un fu- } \\
\text { turo próximo, ¿̇iensa Ud. que si lo ha solicitado, la } \\
\text { ley debería autorizar a los médicos a terminar con } \\
\text { su vida cuando ella lo decida? }\end{array}$ & $\begin{array}{c}\mathrm{N}: 1226 \\
49.4 \%\end{array}$ & $\begin{array}{l}N: 496 \\
20.0 \%\end{array}$ & $\begin{array}{l}\mathrm{N}: 216 \\
8.7 \%\end{array}$ & \multicolumn{2}{|c|}{$14.3 \%$} & $\begin{array}{l}N: 167 \\
6.7 \%\end{array}$ & $\begin{array}{l}\mathrm{N}: 22 \\
0.9 \%\end{array}$ & $\begin{array}{c}\mathrm{N}: 2481 \\
100 \%\end{array}$ & 1 \\
\hline $\begin{array}{l}\text { P.36 Y, si esta persona lo solicita, ¿cree que la ley } \\
\text { debería autorizar a los médicos a recetarle una do- } \\
\text { sis suficiente de fármacos para que ella misma ter- } \\
\text { mine con su vida, tomándoselos cuando lo decida? }\end{array}$ & $\begin{array}{l}\mathrm{N}: 1006 \\
40.5 \%\end{array}$ & $\begin{array}{l}\mathrm{N}: 482 \\
19.4 \%\end{array}$ & $\begin{array}{l}\mathrm{N}: 250 \\
10.1 \%\end{array}$ & \multicolumn{2}{|c|}{$\begin{array}{l}\mathrm{N}: 522 \\
21 \%\end{array}$} & $\begin{array}{l}\mathrm{N}: 192 \\
7.7 \%\end{array}$ & $\begin{array}{l}\mathrm{N}: 29 \\
1.2 \%\end{array}$ & $\begin{array}{c}N: 2481 \\
100 \%\end{array}$ & 1 \\
\hline & (1) & (2) & (3) & (4) & (5) & (6) & (7) & Total & Moda \\
\hline $\begin{array}{l}\text { P.37 ¿Cree Ud. que en España debería regularse } \\
\text { por Ley la eutanasia? }\end{array}$ & $\begin{array}{l}N: 1449 \\
58.4 \%\end{array}$ & $\begin{array}{l}\mathrm{N}: 377 \\
15.2 \%\end{array}$ & $\begin{array}{l}N: 104 \\
4.2 \%\end{array}$ & $\begin{array}{l}\mathrm{N}: 256 \\
10.3 \%\end{array}$ & $\begin{array}{l}\mathrm{N}: 146 \\
5.9 \%\end{array}$ & $\begin{array}{l}\mathrm{N}: 24 \\
1.0 \%\end{array}$ & $\begin{array}{c}\mathrm{N}: 125 \\
5 \%\end{array}$ & $\begin{array}{c}N: 2481 \\
100 \%\end{array}$ & 1 \\
\hline P.38 ¿Y el suicidio médicamente asistido? & $\begin{array}{l}N: 966 \\
38.9 \%\end{array}$ & $\begin{array}{l}\mathrm{N}: 342 \\
13.8 \%\end{array}$ & $\begin{array}{l}\mathrm{N}: 180 \\
7.3 \%\end{array}$ & $\begin{array}{l}\mathrm{N}: 470 \\
18.9 \%\end{array}$ & $\begin{array}{l}N: 180 \\
7.3 \%\end{array}$ & $\begin{array}{l}\mathrm{N}: 20 \\
0.8 \%\end{array}$ & $\begin{array}{l}N: 323 \\
13 \%\end{array}$ & $\begin{array}{l}N: 2481 \\
100 \%\end{array}$ & 1 \\
\hline
\end{tabular}

Fuente: Centro de Investigaciones Sociológicas, España, 2009. Encuesta nacional, 2.481 entrevistas a población mayor de 18 años. Número de Estudio: 2.803.

(1) Sí con toda seguridad

(2) Creo que sí, pero no estoy totalmente seguro

(3) Creo que no, pero no estoy totalmente seguro

(4) No con toda seguridad
(5) No sabe

(6) No contesta

(7) No sabe qué significa
La recodificación de las opciones de respuesta (Tabla III, primera recodificación) en dos categorías (acuerdo vs desacuerdo), muestra cómo en todos los casos los ciudadanos mayoritariamente apoyan las practicas eutanásicas que se plantean variando el grado de acuerdo entre un $52.7 \%$ (P.38) y un $80.5 \%$ (P.24). Las cuestiones donde los ciudadanos alcanzan mayor porcentaje de acuerdo plantean supuestos sobre aceptación y regulación de la eutanasia, mientras que cuando se pregunta por aceptación y regulación de SMA, el grado de acuerdo (aun siendo mayoritario) desciende.

Por último, como se dijo en el apartado de metodología, con objeto de conocer el grado de acuerdo sin ambigüedad de la ciudadanía española, tomamos como postura favorable solo la respuesta "Sí, con toda seguridad" comparándola con la recodificación de todas las demás opciones de respuesta como si fuesen negativas (ver en Tabla III, segunda recodificación) y las sometemos a un contraste mediante la prueba de McNemar (ver Tabla IV). A un nivel de confianza del $99 \%$ las pruebas realizadas permiten afirmar que, 1) existe una actitud más favorable a la regulación de una ley que permita a los médicos ayudar a morir a sus pacientes, que al hecho de no sancionar a los médicos que lleven a cabo dicha acción. 2) En los dos supuestos planteados, enfermedad en fase terminal y enfermedad degenerativa incurable, los ciudadanos se inclinan más por regular y permitir la eutanasia que el SMA. 3) Ambas formas de poner fin a la vida del paciente son más apoyadas cuando este padece una enfermedad en fase terminal, que cuando se trata de una enfermedad degenerativa que no conduce necesariamente a la muerte. 
Tabla III. Recodificación categorías de respuesta

\begin{tabular}{|c|c|c|c|c|}
\hline & \multicolumn{2}{|c|}{ Primera recodificación } & \multicolumn{2}{|c|}{ Segunda recodificación } \\
\hline & $\begin{array}{r}\% \text { de } \\
\text { acuerdo } \\
(1+2)\end{array}$ & $\begin{array}{l}\% \text { en des- } \\
\text { acuerdo } \\
\qquad(3+4)\end{array}$ & $\begin{array}{l}\% \text { acuerdo } \sin \\
\text { ambigüedad } \\
\text { (1) }\end{array}$ & $\begin{array}{l}\% \text { en contra } \\
(2+3+4+5+6)\end{array}$ \\
\hline $\begin{array}{l}\text { P.24 Cuando una persona tiene una enfermedad en fase } \\
\text { terminal, que le causa grandes sufrimientos y que le causa- } \\
\text { rá la muerte en poco tiempo, ¿cree Ud. que la ley debería } \\
\text { permitir que los médicos pudieran poner fin a su vida y a } \\
\text { sus sufrimientos, si esta persona lo solicita libremente? }\end{array}$ & 80.5 & 14.5 & 63.3 & 36.7 \\
\hline $\begin{array}{l}\text { P.25 Y si esta persona lo solicita libremente a sus médicos, } \\
\text { ¿cree Ud. que la ley debería permitir que éstos le propor- } \\
\text { cionasen los medios necesarios para que ella misma pudie- } \\
\text { ra terminar con su vida? }\end{array}$ & 67.9 & 25.3 & 49.7 & 50.3 \\
\hline $\begin{array}{l}\text { P.28 ¿Cree Ud. que debería castigarse al médico que ponga } \\
\text { fin, sin dolor, a la vida de un paciente, en la fase final de } \\
\text { una enfermedad irreversible si éste se lo pide de manera } \\
\text { reiterada y consciente? }\end{array}$ & 14.4 & 75.7 & 7.3 & 92.7 \\
\hline $\begin{array}{l}\text { P.35 En el caso de una persona mayor de } 18 \text { años, con una } \\
\text { enfermedad degenerativa incurable, que le va a incapaci- } \\
\text { tar mental y físicamente en un futuro próximo, ¿piensa Ud. } \\
\text { que si lo ha solicitado, la ley debería autorizar a los médicos } \\
\text { a terminar con su vida cuando ella lo decida? }\end{array}$ & 69.4 & 23.0 & 49.4 & 50.6 \\
\hline $\begin{array}{l}\text { P.36 Y, si esta persona lo solicita, ¿cree que la ley debería } \\
\text { autorizar a los médicos a recetarle una dosis suficiente de } \\
\text { fármacos para que ella misma termine con su vida, tomán- } \\
\text { doselos cuando lo decida? }\end{array}$ & 59.9 & 31.1 & 40.5 & 59.5 \\
\hline $\begin{array}{l}\text { P.37 ¿Cree Ud. que en España debería regularse por Ley la } \\
\text { eutanasia? }\end{array}$ & 73.6 & 14.5 & 58.4 & $41.6^{*}$ \\
\hline P.38 ¿Y el suicidio médicamente asistido? & 52.7 & 26.2 & 38.9 & $61.1^{*}$ \\
\hline
\end{tabular}

Fuente: Elaboración propia a partir de los datos del Centro de Investigaciones Sociológicas, España, 2009. Encuesta nacional, 2.481 entrevistas a población mayor de 18 años. Número de Estudio: 2.803.
(1) Sí con toda seguridad
(5) No sabe
(2) Creo que sí, pero no estoy totalmente seguro
(6) No contesta
(3) Creo que no, pero no estoy totalmente seguro
(7) No sabe qué significa
(4) No con toda seguridad
$*(2+3+4+5+6+7)$ La categoría de respuesta (7), sólo aparece en estas preguntas.

Tabla IV. Prueba McNemar

\begin{tabular}{|c|c|c|c|c|c|c|c|c|c|c|c|c|}
\hline & \multicolumn{2}{|c|}{$\begin{array}{l}\text { Legislar vs } \\
\text { no castigar }\end{array}$} & \multicolumn{2}{|c|}{ Eutanasia vs SMA } & \multicolumn{2}{|c|}{$\begin{array}{l}\text { SMA: enfermedad } \\
\text { terminal vs enferme- } \\
\text { dad degenerativa }\end{array}$} & \multicolumn{2}{|c|}{$\begin{array}{l}\text { E n fe rm e d a d } \\
\text { terminal: euta- } \\
\text { nasia vs SMA }\end{array}$} & \multicolumn{2}{|c|}{$\begin{array}{l}\text { Enfermedad dege- } \\
\text { nerativa: eutana- } \\
\text { sia vs SMA }\end{array}$} & \multicolumn{2}{|c|}{$\begin{array}{l}\text { Eutanasia: enferme- } \\
\text { dad terminal vs enfer- } \\
\text { medad degenerativa }\end{array}$} \\
\hline & P.24 & P.28 & P37 & P.38 & P.24 & P.25 & P.35 & P.36 & P.24 & P.35 & P. 25 & P.36 \\
\hline $\mathbf{P}_{1}^{*}$ & .63 & .59 & .58 & .40 & .63 & .50 & .49 & .41 & .63 & .49 & .50 & .41 \\
\hline $\mathbf{P}^{* *}$ & $p<.001$ & $\mathrm{p}<.001$ & $\mathrm{p}<.001$ & $p<.001$ & $p<.001$ & $p<.001$ & & & & & & \\
\hline
\end{tabular}

*P proporción de "acuerdo sin ambigüedad"

${ }^{* *} p(.99)$ 


\section{DISCUSIÓN}

Vistos los resultados de los análisis parece oportuno comenzar esta discusión comparando el actual marco legislativo español, con el existente en nuestro entorno socioeconómico.

En España el Código Penal (CP) de 1995 castiga con penas de cárcel de 2 a 5 años al que colabore al suicidio de otro (143.2) y de 6 a 10 años si la cooperación llega a ejecutar la muerte (143.3). Si existe "la petición expresa, seria e inequívoca" de la víctima, cuando esta sufre una "enfermedad grave que conduzca a su muerte o que produzca graves padecimientos permanentes y difíciles de soportar" el castigo por cooperar al suicidio ajeno podrá reducirse de 6 meses a 2 años de prisión y de 1 año y 6 meses a 6 años si la cooperación llega a ejecutar la muerte (143.4).

Es interesante subrayar que los atenuantes recogidos en el artículo 143.4 de nuestro CP son prácticamente idénticos a las condiciones exigidas por la normativa de Holanda, Bélgica, Luxemburgo, Washington y Oregón para poder llevar a cabo la práctica de eutanasia y SMA sin cometer delito; ello no es fruto de la casualidad sino más bien de un intenso debate en el legislativo que si bien no llegó a culminar en la despenalización sí que utilizando el recurso de los atenuantes mostraba cierta sensibilidad ante estas situaciones.

Por otro lado, la Ley 41/2002 de 14 de Noviembre, reconoce la autonomía del paciente para decidir sobre las actuaciones en el ámbito de su salud. Además toda persona mayor de edad, capaz y libre puede redactar un documento de instrucciones previas, donde dejar expresada su voluntad sobre cuidados y tratamientos, con el objetivo de que esta se cumpla si se dan situaciones en cuyas circunstancias no sea capaz de expresarlas(Sanz-Ortiz, 2006). Coincide así nuestra normativa, con la holandesa, la belga y la luxemburguesa, al reconocer la validez de este documento como expresión de la voluntad del paciente que debe respetarse. Sin embargo, la voluntad expresada por el paciente no puede contravenir el ordenamiento jurídico, por lo que en nuestro Estado, ante una situación irreversible que causa un sufrimiento intenso, las opciones generales son:
a) Rechazo del tratamiento
b) Limitación del esfuerzo terapéutico
C) Sedación paliativa

Ninguna de estas acciones tiene como objetivo ex- plícito poner fin a la vida del enfermo (Monzón et al. 2008), objetivo que sí alcanzan las prácticas de eutanasia y de SMA. Nuestro ordenamiento jurídico protege la vida, en todos los casos, es decir incluso contra la voluntad del sujeto, sin embargo a la luz de los resultados expuestos más arriba, la sociedad española actual pareciera estar de acuerdo con la posibilidad de no continuarla cuando las condiciones en las que se vive solo abocan a un destino: la muerte apremiante, con dolor y sufrimiento (Chapple et al., 2006).

Ahora bien, el grado de apoyo a este extremo varía en función de las tres diferencias encontradas en las legislaciones existentes: 1) tipo de regulación, 2) práctica permitida y 3) condiciones exigidas para llevarlas a cabo; ello nos permite aproximarnos al modelo legal más coincidente con la opinión de los ciudadanos.

Respecto al primer punto, los ciudadanos españoles muestran su preferencia por que exista una regulación específica en esta materia y no un espacio de impunidad en el que se pueda actuar con albedrío. Este resultado aleja la opinión de la ciudadanía del modelo suizo.

A nuestro juicio un marco legal que regule las prácticas de eutanasia y/o SMA garantiza que ha habido un debate previo reflexionado, donde se han analizado las distintas situaciones, permitiendo dar respuesta a las mismas y asegurando la existencia de controles reguladores (Tomás-Valiente, 2005).

Los ciudadanos muestran también un mayor grado de acuerdo con la legalización de la eutanasia, evidencia que los aleja de los modelos americanos y suizo que despenalizan la ayuda al suicidio y les acerca a Holanda, Luxemburgo y especialmente a Bélgica, cuyo texto legal solo se refiere a la eutanasia. En concreto, en el estudio analizado dos tercios de la población apoya la legalización de la eutanasia, proporción no obstante muy similar a la alcanzada en otros estudios nacionales (Durán, 2004; de Miguel y López, 2006) y bastante más alta que la informada en estudios comparativos a nivel europeo (Cohen et al., 2006).

Por último, respecto a los supuestos bajo los cuales se permite la práctica de eutanasia y/o SMA, encontramos un mayor grado de apoyo cuando se trata de una enfermedad en fase terminal, es decir que conduce al enfermo de forma segura a la muerte, lo que acerca la opinión de los ciudadanos a las legislaciones de Oregón, Washington, Luxemburgo y Bélgica. Pero recordemos que los tres primeros han regulado solo el SMA y no la eutanasia que es la situación de mayor consenso en España, por lo que es el modelo de regulación belga al que más se aproximan 
las opiniones y actitudes de la población española. Si bien es verdad que aunque con menor fuerza la sociedad española también apoya el SMA para casos de enfermedad terminal por lo que sería posible tras un debate serio y sin constricciones que se pudiese recorrer el camino hasta el modelo luxemburgués.

Cotejando la opinión de la ciudadanía con las distintas legislaciones, encontramos que los supuestos en los que se muestra un mayor grado de acuerdo son los que Bélgica adoptó en su legislación: en el ámbito sanitario, promulgó una Ley (legisló frente a no sancionar), que despenaliza la eutanasia (y no el SMA) en casos de enfermedad terminal (aunque de forma laxa contempla otros). Por ello, nuestra propuesta es tomar el modelo belga como marco de referencia a la hora de debatir en profundidad y explícitamente la posibilidad de ampliar los derechos de decisión de las personas sobre su propia vida en nuestro país. A esta misma conclusión han llegado otros autores a partir de aproximaciones racionalistas (Simón y Barrio, 2012) no empíricas.

En España, probablemente aún se demorará el impulso de una Ley que permita decidir sobre la propia muerte, pero ello no debe desalentarnos para desarrollar trabajos empíricos que nos den cuenta de la situación real en la que nos encontramos (Rietjens et al., 2009) si consideramos que son los ciudadanos los que deben orientar el diseño e implementación de las políticas públicas y en general definir el bienestar. De hecho, hay muchos temas que no están suficientemente bien informados o en los que los pocos estudios de los que disponemos muestran contradicciones, por ejemplo: en el estudio de la Organización de Consumidores y Usuarios (OCU) (2000) entre un $65 \%$ de médicos y un $85 \%$ de enfermeras reconoce haber recibido una petición de eutanasia o SMA. Sin embargo, en la encuesta del CIS (2002) se afirma que el $80 \%$ de los médicos jamás ha recibido una petición de eutanasia, y el 91,9\% nunca ha recibido una petición de SMA (Aguiar, Serranodel-Rosal y Sesma, 2009).

En Bélgica la Ley Relativa a la eutanasia, fue votada tras tres años de debate parlamentario y no fue avalada por ninguna asociación médica, sin embargo, en Holanda, el debate público sobre la eutanasia y el SMA se prolongó durante 30 años hasta llegar a la promulgación de una Ley (Vermeersch, 2002; Deliens y van der Wal, 2003). En nuestro país, el debate es llevado a la Cámara del Congreso en los años 90 y desde entonces y hasta ahora varias iniciativas se han sucedido, siendo siempre rechazadas. Además, como ya hemos señalado, diversas encuestas, muestran el apoyo mayoritario de la sociedad hacia la regulación de la eutanasia. Y más aún, el comité consultivo de ética de Cataluña, (formado por médicos, juristas, filósofos) órgano asesor de la Generalitat, ya en el año 2007 presentó un informe a petición de la Consejería de Salud, en el que abogaba por la permisión de la eutanasia en enfermos terminales o con patologías irreversibles, pidiendo que se reformara el Código Penal (Armengol et al. 2006).

Los avances legislativos que se han conseguido en nuestro país, reconociendo mayor autonomía al paciente en la toma de decisiones sobre los procesos que implican su salud, deberían ir acompañados de una reflexión más profunda sobre las opciones al final de la vida. No debemos ocultar este debate ni olvidar reflexionar e investigar sobre cómo la muerte está teniendo lugar en nuestros días. Acumular trabajos de investigación que aborden estas cuestiones, permitirá una base sólida sobre la que poder construir el debate más allá de juicios morales personales llegando a una regulación de consenso que ampare legalmente nuevas prácticas al final de la vida y, en general, nuestros derechos de salida.

\section{NOTA ACLARATORIA}

El análisis presentado en este trabajo se realizó sobre la Ley Belga "Relativa a la eutanasia" aprobada en 2002. Esta Ley ha sido revisada en 2014 para extender la eutanasia a los menores. Este extremo no es considerado en el presente documento por ser posterior a su realización.

\section{AGRADECIMIENTOS}

Al Fondo Social Europeo por la cofinanciación del Contrato JAE-Doc del Programa "Junta para la Ampliación de Estudios" que ha hecho posible el desarrollo de este trabajo. 


\section{BIBLIOGRAFÍA}

Aguiar González, F.; Serrano-del-Rosal, R. y Sesma, D. (2009). Eutanasia y suicidio asistido: un debate necesario. Policy papers, 3.

Armengol i Millans, R.; Boladeras CucureIla, M.; Broggi i Trias, M. A.; Camps Cervera, V.; Espasa i Oliver, R.; Hernández García, J.; Morlans, M. y Pérez Oliva, M. (2006). Informe sobre l'eutanàsia $i$ l'ajuda al suïcidi. Informe sobre la eutanasia y la ayuda al suicidio. Barcelona: Generalitat de Catalunya.

Chapple, A.; Ziebland, S.; McPherson, A. y Herxheimer, A. (2006). What people close to death say about euthanasia and assisted suicide: a qualitative study. Journal of Medical Ethics, 32, 12, pp. 706-710. http://dx.doi.org/10.1136/ jme.2006.015883

Cohen, J.; Marcoux, I.; Bilsen, J.; Deboosere, P.; van der Wal, G. y Deliens, L. (2006). European public acceptance of euthanasia: socio-demographic and cultural factors associated with the acceptance of euthanasia in 33 European countries. Social Science \& Medicine, 63, 3, pp. 743-756. http://dx.doi. org/10.1016/j.socscimed.2006.01.026

De Miguel Sánchez, C.; López Romero, A (2006). Eutanasia y suicidio asistido. Conceptos generales, situación legal en Europa, Oregón y Australia (I), Medicina Paliativa, 13, 4, pp. 207-215

Deliens, L. y van der Wal, G. (2003). The euthanasia law in Belgium and the
Netherlands, The Lancet, 362, pp. 1239-1240.

Durán Heras, M. A. (2004). La calidad de muerte como componente de la calidad de vida. Revista Española de Investigaciones Sociológicas, 106, pp. 9-32. http://dx.doi.org/10.2307/40184583

Durán Heras, M.A. (2007). Los derechos de salida. En: Aramayo, R. y Auxin, T. Interdependencia. El bienestar como requisito de la dignidad [CD-ROM] San Sebastián: II Encuentro de Moral, Ciencia y Sociedad.

Herrera Gómez, M. (2001) Las políticas sociales en el Welfare Mix. Revista Española de Investigaciones Sociológicas, 96, pp. 71-94. http://dx.doi. org/10.2307/40184384

Ministerio de Sanidad, Política Social e Igualdad (2010). Plan de Calidad para el Sistema Nacional de Salud. Recuperado de http://www.mspsi.es/organizacion/ sns/planCalidadSNS/pdf/pncalidad/ PlanCalidad2010.pdf

Monzón Marín, J. L.; Saralegui Reta, I.; Abizanda i Campos, R.; Cabré Pericas, L.; Iribarren Diarasarri, S.; Martín Delgado, M. C. y Martínez Urionabarrenetxea, K. (2008). Recomendaciones de tratamiento al final de la vida del paciente crítico. Medicina intensiva, 32, 3, pp. 121-133. http://dx.doi.org/10.1016/ S0210-5691(08)70922-7

Rietjens, J. A. C.; van der Maas, P. J.; Onwuteaka-Philipsen, B. D.; van Delden, J.
J.; y van der Heide, A. (2009). Two decades of research on euthanasia from the Netherlands. What have we learnt and what questions remain. Journal of Bioethical Inquiry, 6, 3, pp. 271-283. http://dx.doi.org/10.1007/s11673009-9172-3

Royes, A. (2008). La eutanasia y el suicidio médicamente asistido. Psicooncología, 5, 2-3, pp. 323-337.

Sanz-Ortiz, J. (2006). ¿Es posible gestionar el proceso de morir? Voluntades anticipadas. Medicina Clínica, 126, 16, pp. 620-623. http://dx.doi. org/10.1157/13087720

Simón Lorda, P. y Barrio Cantalejo, I. (2012). La eutanasia en Bélgica. Revista Española de Salud Pública, 86, 1, pp. 5-19. http://dx.doi.org/10.1590/S113557272012000100002

Tomás-Valiente Lanuza, C. (2005). Posibilidades de regulación de la eutanasia solicitada. Fundación Alternativas. Documento de Trabajo 71/2005. Recuperado de http://www.falternativas.org/laboratorio/documentos/documentos-detrabajo/posibilidades-de-regulacionde-la-eutanasia-solicitada

Vermeersch, E. (2002). The Belgian Law on Euthanasia. The Historical and Ethical Background. Acta Chirurgica Belga, 102, 6, pp. 394-397. 